\title{
A Cooperative Spectrum Sensing Information Transmission Scheme Based on Cyclic Delay Diversity
}

\author{
Yanru LIU \\ Key Lab of Intelligent Computing and \\ Signal Processing of Ministry of \\ Education, Anhui University \\ Hefei, China
}

\author{
Dechao HU \\ Key Lab of Intelligent Computing and \\ Signal Processing of Ministry of \\ Education, Anhui University \\ Hefei, China
}

\author{
Xiaohui LI* \\ Key Lab of Intelligent Computing and \\ Signal Processing of Ministry of \\ Education, Anhui University \\ Hefei, China
}

\begin{abstract}
To improve the reliability of spectrum sensing, a cooperative spectrum sensing information transmission scheme is discussed in this paper. Pre-coding and cyclic delay diversity (CDD) techniques are fully considered and properly combined to obtain diversity gains. Simulation results show that the proposed scheme can reduce error rate and further improve the cooperative spectrum sensing performance in the multipath fading channel.
\end{abstract}

Keywords-Cooperative spectrum; Cyclic delay diversity; Spacefrequency coding

\section{INTRODUCTION}

In order to deal with the imbalance between spectrum under-utilization and spectrum scarcity, cognitive radio(CR)has been proposed [1]. By sensing and adapting to the environment, $\mathrm{CR}$ is able to operate in spectrum holes and serve its users to avoid harmful interference to the primary users (PU).

To improve the performance of spectrum sensing, cooperation amongst the secondary users (SUs) has been proposed [2]. In the cooperative spectrum sensing system, sensing information should be transmitted to the fusion center after it is accurately detected. Due to the fading, decision received from some cooperative CR users may be received erroneously at fusion center (FC), transmission efficiency is greatly reduced and the reliability of cooperative spectrum sensing system is low [3-4].

Space-frequency coding scheme based on cyclic delay diversity is researched [5-6] because its design is simple and it has a robust. However, early schemes suffered very low spectrum efficiency and diversity gains. To overcome this shortcoming, Witrisal [7] developed a method using CDD in Multiple Input Multiple Output and Orthogonal Frequency Division Multiplexing (MIMO-OFDM) system. This method can obtain highly frequency diversity gain. But this frequency diversity is obtained through error correction coding technology .The technology cause high detection complexity.

In this paper, we propose a cooperative spectrum sensing information transmission scheme. Pre-coding and cyclic delay diversity (CDD) techniques are fully considered and properly combined. Simulation results show that the proposed scheme can reduce error rate and improve the cooperative spectrum sensing performance in the multipath fading channel. False alarm probability is reduced and detection probability is increased, at the same time, the reliability of spectrum sensing is also improved.

\section{COOPERATIVE SPECTRUM SENSING MODEL}

We consider a CR network (CRN) composed of M CR users (CRUs) and a FC which make a final decision, as shown in Fig.1.

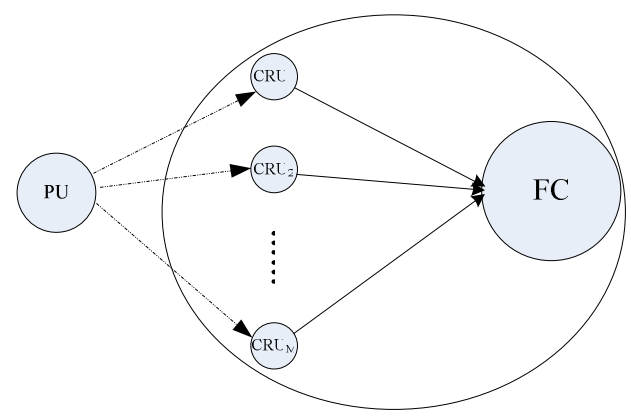

Fig.1 Cognitive radio network

In general, cooperative spectrum sensing is performed as follows[6]:

Step 1: Every CRU performs local spectrum measurements independently and make a binary decision;

Step 2: All of the CRUs forward their binary decisions to the FC;

Step 3: The FC combines those binary decisions and makes a final decision based on fusion decision criterion to infer the absence or presence of the PU in the observed frequency band. 
The ultimate goal of spectrum sensing is to decide the presence of PU, the basic model for spectrum sensing by the CRU, which is defined as

$$
r(t)=\left\{\begin{array}{cc}
h s(t)+n(t) & H_{1} \\
n(t) & H_{0}
\end{array}\right.
$$

where $r(t)$ is the signal received by CRU, $s(t)$ is the transmitted signal of the PU, $n(t)$ is additive white Gaussian noise (AWGN) and $h$ is the amplitude gain of the channel. $H_{1}$ indicates the presence of PU whereas $H_{0}$ indicates only noise.

The output of energy detector has the distribution defined as follows:

$$
Y=\left\{\begin{array}{cc}
\chi_{2 N}^{2}(2 \rho) & H_{1} \\
\chi_{2 N}^{2} & H_{0}
\end{array}\right.
$$

where $\chi_{2 N}^{2}$ and $\chi_{2 N}^{2}(2 \rho)$ represent central chi-square distribution and non-square distribution with $2 N$ degree of freedom and non centrality parameter, $2 \rho$ is for latter distribution.

The energy value is then compared with the predetermined threshold $\lambda$. Each CRU gets the first decision locally as

$$
\text { Decision }=\left\{\begin{array}{l}
1, Y>\lambda \\
0, Y<\lambda
\end{array}\right.
$$

\section{CODING SCHEME}

In the CRN system, the spectrum hole is sought by CRUs, and then the communication among CRUs is conducted using the spectrum holes. The communication must be not cause harmful interference to PU, therefore, CRUs must uninterrupted detect the PU signal and make a decision independent, then the decision results are transmitted to the fusion center. Once PU signal is detected, CRUs must exit current spectrum immediately, and then new spectrum hole is searched. This constitutes a process which the sensing information is transmitted to the fusion center successionally.

Every CRU only get their own information, after information exchange is filled on, every CRU have all information of the other CRUs. The sensing information set is defined as $\left\{\begin{array}{llll}u_{1} & u_{2} & \cdots & u_{M}\end{array}\right\}$. After information is exchanged, the stream of source signal can be defined as:

$$
\begin{array}{llllllll}
u_{1}(1) & u_{2}(1) & \cdots & u_{M}(1) & u_{1}(2) & u_{2}(2) & \cdots & u_{M}(2) \\
\cdots & u_{m}(t) & \cdots
\end{array}
$$

where $u_{m}(t)$ is the sensing information of the m-th CRU in tth time slots.

The error rate may be increased because of the correlation between the sub-carriers. Therefore space-frequency transmission matrix must be divided into groups in all subcarriers firstly. $L$ is the length of impulse response, $N_{F}$ is the total number of sub-carriers. All sub-carriers can be divided into $G$ groups, where

$$
G=\left\lfloor N_{F} / M L\right\rfloor .
$$

Space-frequency mapping matrix $S_{g}$ is defined as:

$$
S_{g}=\left[S_{g}^{0} S_{g}^{1} \cdots S_{g}^{L-1}\right], g \in[0, G-1]
$$

$S_{g}^{l}$ is given as follows.

$$
S_{g}^{l}=\left[\begin{array}{ccccc}
U[(G l+g) M+1] & U[(G l+g) M+2] & \cdots & U[(G l+g) M+M] \\
U[(G l+g) M+2] & U[(G l+g) M+3] & \cdots & U[(G l+g) M+1] \\
\vdots & \vdots & \ddots & \vdots \\
U[(G l+g) M+M] & U[(G l+g) M+1] & \cdots & U[(G l+g) M+M-1]
\end{array}\right]
$$

where the $U$ is the modulated symbols of source signal, $U[k]$ is the $\mathrm{k}$-th modulated symbol.

Using pre-coding technique, encoding matrix $X_{g}$ is got and can be defined as follows.

$$
X_{g}=\left[X_{g}^{0} X_{g}^{1} \cdots X_{g}^{L-1}\right], \quad g \in[0, G-1]
$$

where

$$
\begin{array}{r}
X_{g}^{l}(m, i)=\Theta_{l+1} \cdot\left[S_{g}^{0}(m, i) S_{g}^{1}(m, i) \cdots S_{g}^{L-1}(m, i)\right]^{T}, \\
i \in[1, M], m \in[1, M], l \in[0, L-1]
\end{array}
$$

$\Theta$ is a $L \times L$ pre-coding matrix, $\Theta_{l+1}$ is $(l+1)$-th line of $\Theta$.

Spatial diversity and frequency diversity can be obtained utilizing pre-coding technique within each sub-carrier group while the spectrum utilization rate is unchanged.

\section{TRANSMISSION MODEL}

Orthogonal frequency-division multiplexing (OFDM) technology and CDD operations are used to complete transmission and the transmission model of space-frequency coding block at present can be expressed as (8)

$$
\begin{aligned}
& Y((G l+g) M+i) \\
& =\sum_{m=1}^{M}\left(H_{m}((G l+g) M+i)\left(X_{g}^{l}\right)^{C D D}(m, i)\right)+W((G l+g) M+i)
\end{aligned}
$$


where

$$
\begin{aligned}
\left(X_{g}^{l}\right)^{C D D}(m, i) & =e^{-j \frac{2 \pi}{N_{F}}((G l+g) M+i) \Delta_{m}} \cdot X_{g}^{l}(m, i) \\
g & \in[0, G-1], \quad i \in[1, M], \\
m & \in[1, M], \quad l \in[0, L-1]
\end{aligned}
$$

$\Delta_{m}$ is the cyclic delay value for m-th CR user.

Make

$$
\begin{gathered}
H^{C D D}(g, l, i)=\left[\begin{array}{c}
H_{1}((G l+g) M+i) e^{-j \frac{2 \pi}{N_{F}}((G l+g) M+i) \Delta_{1}} \\
H_{2}((G l+g) M+i) e^{-j \frac{2 \pi}{N_{F}}((G l+g) M+i) \Delta_{2}} \\
\vdots \\
H_{M}((G l+g) M+i) e^{-j \frac{2 \pi}{N_{F}}((G l+g) M+i) \Delta_{M}}
\end{array}\right]^{T} \\
{\left[X_{g}^{l}(1, i) X_{g}^{l}(2, i) \cdots X_{g}^{l}(M, i)\right]^{T}=A(i) \cdot\left[X_{g}^{l}(1,1) X_{g}^{l}(2,1) \cdots X_{g}^{l}(M, 1)\right]^{T}} \\
A(i)=\left[\begin{array}{ll}
0 & I_{M-i+1} \\
I_{i-1} & 0
\end{array}\right] \\
\tilde{Y}(g, l, i)=Y((G l+g) M+i) \\
\tilde{W}(g, l, i)=W((G l+g) M+i)
\end{gathered}
$$

where $H^{C D D}(g, l, i)$ is the equivalent channel matrix when coding matrix is transmitted, $\tilde{Y}(g, l, i)$ is the received matrix and $\tilde{W}(g, l, i)$ is additive complex Gaussian noises matrix.

The whole transmitted model can be simplified as follows.

$$
\begin{aligned}
& \tilde{Y}(g, l, i) \\
& =H^{C D D}(g, l, i) \cdot A(i) \cdot\left[X_{g}^{l}(1,1) X_{g}^{l}(2,1) \cdots X_{g}^{l}(M, 1)\right]^{T}+\tilde{W}(g, l, i)
\end{aligned}
$$

\section{SIMULATION RESULTS}

To test the bit error performance of the proposed scheme is simulated. We assume the number of CRU is 3 in this system and the criterion is Chair-Varshney in fusion center. The channels between the CRU and fusion center are established with unit variance according to the time-frequency double selective fading models in this paper. QPSK modulation is adopted, and all modulated symbols are transmitted on proper sub-carriers of the wireless channels. The number of subcarriers is 64, the carrier frequency is $3.5 \mathrm{GHZ}$, and the path number of all multi-path channels is set as 2 .

The performances are tested in turn and the simulated results are compiled in Fig. 2. Based on Pre-coding in frequency domain, the probability of false alarm and the probability of miss detection are reduced, and the reliability of the system is enhanced.And it also shows that the result is the same as the results above if only the average of the group delay distortion is considered. That is the more serious distortion of group delay, the greater deterioration of BER performance.

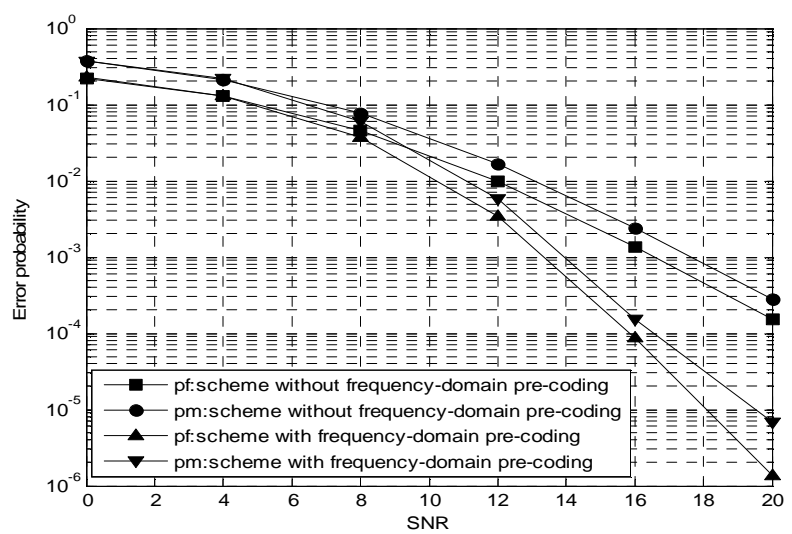

Fig. 2: Error probability comparisons with pre-coding or not in subcarrier group

Next, further simulations are completed in Fig. 3. In simulation when CDD delay is used, the performance of the system is improved. The reliability of the system is increased greatly when SNR is more than $12 \mathrm{~dB}$.

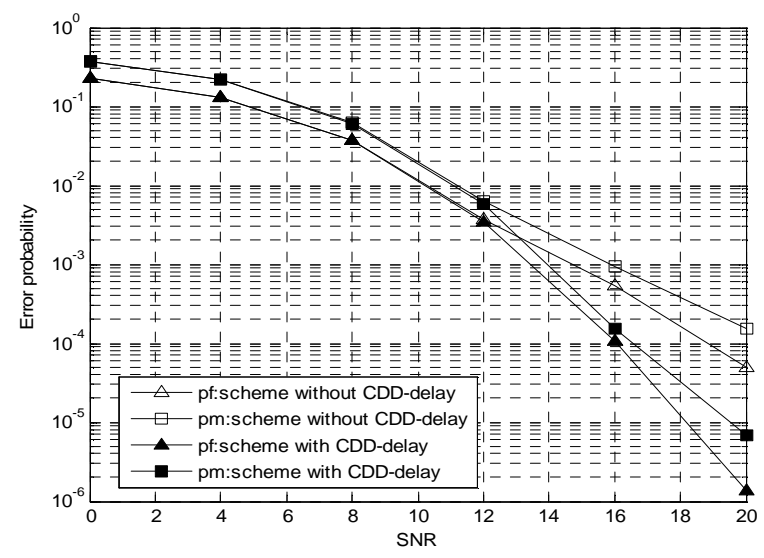

Fig. 3: Error probability comparisons with CDD delay or not in subcarrier group

\section{CONCLUSION}

This paper mainly studies the cooperative spectrum sensing information transmission scheme based on cyclic diversity. This scheme can solve the problem of high error rate in the multipath fading channel and obtain diversity gain of the whole system.

\section{ACKNOWLEDGMENT}

This project is supported by the National Natural Science Foundation of China (No. 60972040), the Anhui Provincial 
Natural Science Foundation (No. 11040606Q06), and the Provincial Project of Natural Science Research for Colleges and Universities of Anhui Province of China (No. KJ2012A003).

\section{REFERENCE}

[1] Haykin S., Thomson D. J., Reed J. H., "Spectrum sensing for cognitive radio"Proceedings of the IEEE, 2009,97(5):849-877.

[2] Chen Q., Motani M., Wong W., Nallanathan A., "Cooperative spectrum sensing strategies for cognitive radio mesh networks", IEEE Journal of Selected Topics in Signal Processing, 2011,5(1):56-67.

[3] A. Ghasemi and E. S. Sousa, "Collaborative spectrum sensing for opportunistic access in fading environments," in Proc. 1st IEEE Symp. New Frontiers in Dynamic Spectrum Access Networks, Baltimore, USA, Nov. 8-11, 2005, pp. 131-136.
[4] Michalopoulos D. S., Lioumpas A. S., Karagiannidis G. K., Schober R., "Selective cooperative relaying over timevarying channels", IEEE Transactions on Communications, 2010,58(8):2402-2412.

[5] Bauch G., Malik, J.S., Orthogonal frequency division multiple access with cyclic delay diversity, in ITG Workshop on Smart Antennas, Munich, Germany, 2004:17-24.

[6] Tan J., Stuber G. L., Multicarrier delay diversity modulation for MIMO systems, IEEE Transactions on Wireless Communications, 2004, 3(5):1756-1763.

[7] Witrisal K., Kim Y.-H., Prasad R., et al., Antenna diversity for OFDM using cyclic delays, In SCVT-2001, 2001, 13-17.

[8] Siyang Liu, Feifei Wang, Ranran Zhang, Yachen Wang, and Yuanan Liu. Full Diversity Spreading Code for Downlink Spaee-Time-Frequeney Spreading CDMA, in proc. IEEE ICC’08, Beijing, China, May 2008:5048-5052. 